\title{
Occurence of Internet Addiction in a General Population Sample: A Latent Class Analysis
}

\author{
Hans-Jürgen Rumpf ${ }^{a} \quad$ Ad A. Vermulst $^{c}$ Anja Bischof ${ }^{a}$ Nadin Kastirke $^{b}$ \\ Diana Gürtler $^{b}$ Gallus Bischof ${ }^{a}$ Gert-Jan Meerkerk ${ }^{d}$ Ulrich John ${ }^{b}$ \\ Christian Meyer ${ }^{\text {b }}$ \\ a Department of Psychiatry and Psychotherapy, University of Lübeck, Research Group S:TEP, Lübeck, \\ ${ }^{b}$ University Medicine Greifswald, Institute of Social Medicine and Prevention, Greifswald, Germany; ${ }^{\circ}$ Department of \\ Developmental Psychopathology, Behavioural Science Institute, Radboud University Nijmegen, Nijmegen, and ${ }^{d}$ IVO \\ Addiction Research Institute, Rotterdam, The Netherlands
}

\section{Key Words}

Internet addiction - General population - Compulsive internet use scale $\cdot$ Latent class analysis . Prevalence

\begin{abstract}
Background: Prevalence studies of Internet addiction in the general population are rare. In addition, a lack of approved criteria hampers estimation of its occurrence. Aims: This study conducted a latent class analysis (LCA) in a large general population sample to estimate prevalence. Methods: A telephone survey was conducted based on a random digit dialling procedure including landline telephone $(n=14,022)$ and cell phone numbers $(n=1,001)$ in participants aged 14 64. The Compulsive Internet Use Scale (CIUS) served as the basis for a LCA used to look for subgroups representing participants with Internet addiction or at-risk use. CIUS was given to participants reporting to use the Internet for private purposes at least $1 \mathrm{~h}$ on a typical weekday or at least $1 \mathrm{~h}$ on a day at the weekend $(n=8,130)$. Results: A 6-class model showed best model fit and included two groups likely to represent Internet addiction and at-risk Internet use. Both groups showed less social participation and the Internet ad-
\end{abstract}

diction group less general trust in other people. Proportions of probable Internet addiction were $1.0 \%$ ( $\mathrm{Cl} 0.9-1.2)$ among the entire sample, $2.4 \%(\mathrm{Cl} 1.9-3.1)$ in the age group 14-24, and $4.0 \%(\mathrm{Cl} 2.7-5.7)$ in the age group 14-16. No difference in estimated proportions between males and females was found. Unemployment (OR 3.13; $\mathrm{Cl} 1.74-5.65)$ and migration background (OR 3.04; $\mathrm{Cl} 2.12-4.36$ ) were related to Internet addiction. Conclusions: This LCA-based study differentiated groups likely to have Internet addiction and at-risk use in the general population and provides characteristics to further define this rather new disorder. @ $2013 \mathrm{~S}$. Karger AG, Basel

\section{Introduction}

There is a quickly growing body of research on problematic use of the Internet. Although generally accepted that formal diagnostic criteria are missing, the existence of compulsive Internet use or Internet addiction is widely claimed and included as a condition for further research in the fifth edition of the Diagnostic and Statistical Manual of Mental Disorders (DSM-5) [1,2]. Different Internet

\section{KARGER}

E-Mail karger@karger.com

www.karger.com/ear
(C) 2013 S. Karger AG, Basel

1022-6877/13/0204-0159\$38.00/0
PD Dr. Hans-Jürgen Rumpf

Department of Psychiatry and Psychotherapy University of Lübeck, Research Group S:TEP

Ratzeburger Allee 160, DE-23538 Lübeck (Germany)

E-Mail hans-juergen.rumpf@uksh.de 
applications are discussed to lead to addiction such as online gaming, social networks or erotica/pornography [36]. However, in DSM-5, the proposed criteria are restricted to Internet gaming. Despite the lack of a consensus concerning diagnostic criteria so far, several studies have tried to estimate the prevalence of Internet addiction. Rates range from 1 to 14\% [7] and a new 21st century epidemic has been discussed [8]. Although in general, higher rates can be found in Asian than in other countries [e.g. 9], regional differences cannot account for the immense difference in prevalence rates that have been found. Two reasons have to be considered: (1) a sample selection bias due to convenience samples and (2) different assessment instruments and criteria to define Internet addiction.

Sample selection bias is very likely to be a major cause of divergent prevalence estimates. The majority of studies have used convenience samples consisting of non-representative subgroups such as can be found in online surveys. In these studies, probability of study inclusion was obviously likely to be confounded with the problem behaviour to be measured. Other studies covered only specific age groups (e.g. students). Such approaches tend to lead to overestimation. Only two studies used general population samples. A telephone survey was conducted in the US general population 18 years or older $(n=2,513$; response rate $56.3 \%$ ). Cellular phone numbers were not included suggesting a coverage bias to be likely. In order to assess Internet addiction, 12 Internet-related questions were used covering aspects of criteria for other impulse control disorders, obsessive-compulsive disorder, substance use disorder and from suggested criteria for problematic Internet use. Four sets of possible diagnostic criteria were used resulting in respective prevalence rates between of 0.3 und $0.7 \%$ [10].

A second general population study from Norway used a stratified probability sample among 16- to 74-year-old individuals [11]. Of 10,000 residents addressed, 3,399 returned a mailed questionnaire or completed it online. The 8-item Young Diagnostic Questionnaire [12] was used. Of the sample, $1 \%$ fulfilled 5 or more criteria and were considered as Internet addicted, an additional 5.2\% were at-risk Internet users (3-4 criteria). Both general population studies suffer from diagnostic measures that have not been sufficiently validated.

Different approaches exist to tap Internet addiction [13]. The most widely used questionnaire is the 20 -item Internet Addiction Test (IAT) [14]. This scale has been developed in clinical settings and psychometric properties for general population samples are unclear. Other assessment instruments such as the Chen Internet Addic- tion Scale (CIAS) [15] have been developed. In addition, criteria supposed to constitute Internet addiction have been proposed $[7,16]$. Proposals cover criteria derived from classifications of substance dependence or pathological gambling and include aspects such as loss of control, withdrawal, preoccupation, loss of interests or activities other than Internet use, continued use despite knowledge of negative consequences and use to escape from negative affective states. Besides these criteria, Tao et al. [7] define a minimum amount of $6 \mathrm{~h} /$ day spent in the Internet to fulfil the diagnosis of Internet addiction. Data to define Internet addiction are derived from clinical settings but may not cover the whole spectrum of characteristics found in the general population.

Besides defining Internet addiction, several studies make additional use of classifications such as problematic Internet use or at-risk use in order to describe less severe conditions with some problems but no full-blown addiction. Clear distinctions between this group and those having Internet addiction are often missing. In the present study, we will use the terms Internet addiction and at-risk Internet use.

Facing the dearth of a consensus concerning criteria to define and tools to assess Internet addiction, a promising solution is to look within samples for latent classes that are likely to be addicted to the Internet. Latent class analysis (LCA) is a statistical method that identifies groups of respondents defined by similarity of response patterns and having a specific probability to belong to a latent class of respondents [17]. The advantage of such a methodological approach is that no a priori definition of the disorder (e.g. number of criteria fulfilled) is necessary. Such an empirical, data-driven approach was able to define subgroups of individuals who are likely to suffer from Internet game addiction or heavy use from a large sample of Dutch school children aged 13-16 years [18]. In two large school-based samples, respondents filled out the Compulsive Internet Use Scale (CIUS) [19], a 14-item questionnaire covering core criteria of problematic Internet use. A LCA revealed a 6-class model to have the best fit. According to the data, 2 of these classes showed characteristics that made it very likely that participants were experiencing heavy use or problems with online gaming: one group showed a high total amount of hours spent online and medium CIUS sum scores representing heavy users and a second group showed high total hours online and as well high CIUS sum scores representing dependent users. This latter group constituted $3 \%$ of the sample and about $1.5 \%$ of all children aged 13-16 years in the Netherlands. However, results are restricted to adolescents and to online gaming. 
In summary, studies on the occurrence of Internet addiction in the general population are rare and suffer from methodological shortcomings. Only two studies used a general population approach, however results are restricted due to poorly validated instruments, rather small sample sizes and a covering bias. Studies aiming to characterize Internet addiction and proposing diagnostic criteria come from clinical settings, however general population data are necessary to cover the full spectrum of the disorder. Considering the absence of a consensus how to define Internet addiction and the lack of populationbased diagnostic research in this area, a data-driven empirical approach using LCA as described above seems to be a promising pathway to improve findings. Following this approach, the present study performed a LCA in a large representative general population sample in order to (a) find subgroups representing Internet addiction and at-risk use, (b) describe characteristics of these groups, (c) estimate the proportions of these groups in the general population, and (d) investigate if the criterion of $6 \mathrm{~h} /$ day [7] spent online is useful in a general population setting.

\section{Method}

The present analysis is based on a sample from the study 'Pathological Gambling and Epidemiology (PAGE)'. PAGE was funded by the German federal states. The present analysis was funded by the German Federal Ministry of Health within the project Prevalence of Internet Addiction (PINTA). PAGE was approved by the ethics committees of the Universities of Greifswald and Lübeck.

PAGE used a patched-up design in recruiting problem and pathological gamblers. One approach consisted of a representative telephone survey which is the basis for the PINTA analysis.

\section{Recruitment}

The telephone survey was conducted in cooperation with 'infas Institut für angewandte Sozialwissenschaft $\mathrm{GmbH}$ ', an institute for social research. The survey was based on a German landline telephone sample and an additional mobile-only sample to cover the growing percentage of individuals that are only accessible via cellular phone numbers.

For the landline telephone survey a clustered and stratified sample was drawn. At the first sampling stage, 53 sample points (52 municipalities, Berlin was represented by two sample points) were selected based on a 'probability proportional to size' procedure. Implicit stratification ensures a representative selection of German federal states, administrative districts and counties. The sample points represent the primary sampling units. At the second stage a fixed number of telephone numbers in each region was generated or selected from available telephone registries. In households reached by random last digit dialing, respondents were included if they belonged to the age group 14-64. In case of more than one person in the respective age group the one who had his birthday at last was chosen (third sampling stage).

Internet Addiction in General Population
In the mobile-only sample, a random selection of nationwide cell phone numbers was used and a screening to include only those who could not be reached via landline telephone was performed. Estimates for Germany suggest that approximately $12 \%$ of the total population are reachable via cell phone but not landline telephone [20]. Mobile-only access was more prevalent among single households and younger people. At the time of data collection for the present study, the prevalence of mobile-only access was estimated at $14 \%$ for the population aged 14-64 years (analyses based on unpublished survey data provided by the Institute for Applied Social Science, infas).

\section{Sample}

The landline sample included 26,736 eligible target persons. Among them, 38.9\% refused to participate, $4.6 \%$ could not be reached because the contacted person refused access to the target person, $2.7 \%$ could not be reached due to other reasons, $1.4 \%$ were too ill to participate, and $52.4 \%$ (14,022 respondents) participated in the interview.

For the mobile-only sample, 1,000 participants were targeted to have sufficient statistical power. To reach this goal, 135,232 phone numbers were contacted of whom 74,058 were invalid and 28,626 could not be contacted. In total, 13,273 valid telephone numbers were screened for eligibility, 11,506 did not fulfil the inclusion criteria (age group 14-46), and 1,767 were eligible target persons. Among them, $0.7 \%$ could not be interviewed due to insufficient German language skills and $0.4 \%$ were too ill or handicapped to participate. In addition, $42.3 \%$ refused to participate, and $56.6 \%$ (1,001 respondents) participated in the interview.

The total sample consisted of $55.2 \%$ females, mean age was 39.9 years (SD 14.0), $15.9 \%$ had 9 years or less of schooling, $31.8 \%$ 10 years, $47.3 \%$ more than 10 years, $4.9 \%$ were still in school, in $0.1 \%$ information was not transferable to years of schooling.

Of the total sample of 15,023 participants, 8,132 reported using the Internet for private purposes at least $1 \mathrm{~h}$ on a typical weekday or at least $1 \mathrm{~h}$ on a day at the weekend. CIUS was assessed in this subgroup. Of those, $3.2 \%$ did not answer to all CIUS items. In the majority of these cases the participants had only one (2.5\%) or two $(0.4 \%)$ missing values. Further, $0.3 \%$ did not answer between 3 and 10 CIUS items. A minimum number of 8 items answered was defined and missing values were replaced by mean values.

\section{Measures}

Internet Addiction

To cover criteria of Internet addiction, the CIUS [19] was applied. This instrument was chosen because data on its psychometric performance in representative samples are available [19]. It consists of 14 items and was developed to assess the following 5 core criteria for Internet addiction: salience, withdrawal, loss of control, conflict and coping with unpleasant mood. Questions are answered on a 5-point Likert-type scale ranging from 'never' to 'very often'. A stable one-factor solution was found across time and different samples [19]. The questionnaire showed good validity and a Cronbach's $\alpha$ as measure of reliability ranging from 0.88 to $0.90[18,19]$. In the current sample, $\alpha$ was 0.87 which approves the good internal consistency of this instrument. No empirically derived cut-off has been published in English yet, however a cut-off score of 28 was suggested from the developers of this instrument [19]. The CIUS items were transformed into German by procedures of translation and back-translation. 
Internet Use

Hours of Internet use were asked with two items: (1) number of hours spent in the Internet for private purposes on a typical weekday and (2) number of hours spent in the Internet for private purposes on a typical day at the weekend. Hours were summed up for a total amount of hours/week. Respondents were asked if there is an Internet activity for private purposes they use for more than $50 \%$ of the time. If participants named more than one main activity, the first one was chosen.

\section{Social Participation and Trust}

Social capital has been identified as an important concept in economic fields and in public health [21]. Two components of social capital are social participation and generalized trust. Social participation is characterized by activities in formal and informal networks. It was assessed with a standardized instrument by asking if respondents had taken part in activities in 13 domains during the last 12 months [22]: study circle/course at work, study circle/course in spare time, union meeting, meeting of an association, theatre/cinema visit, art exhibition, church, public sports event, letter to newspaper/magazine, demonstration of some sort, public entertainment (e.g. night club, dance, etc.), large family meeting, and party at someone's home. Good test-retest reliability was found with a $\kappa$ of 0.70 based on dichotomous results [22]. Generalized trust to other people was assessed with the following item: 'Generally, you can trust other people' with the answer formats 'Do not agree at all', 'Do not agree', 'Agree', and 'Completely agree' [21]. Social participation and trust are hypothesized to be low in individuals with Internet addiction. One of the criteria included in the DSM-5 proposal for Internet gaming disorder is giving up social or recreational activities [2].

\section{Statistical Analysis}

LCA provides classification of individuals and is a special case of finite mixture modeling [23]. Unlike other approaches such as cluster analysis, cases are not absolutely assigned to classes but have a certain probability to belong to a class. LCA was computed using Mplus 5.1 [23] and was used in an exploratory way to find subgroups that represent Internet users with at-risk Internet use or Internet addiction.

There are several criteria to decide on the number of classes [24]. One is the Bayesian Information Criterion (BIC) value. This is a relative measure where a lower BIC value is better. The second one is the entropy measure where high values are preferred. The maximum value is 1 . Currently there are no statistical criteria to decide what is low or high. This measure is a combination of the posterior probabilities. Posterior probabilities denote how well the respondents are classified into their class. Furthermore, the $\mathrm{Vu}$ ong-Lo-Mendell-Rubin likelihood ratio test (VLMR-LRT) and the adjusted Lo-Mendell-Rubin likelihood ratio test (LMR-aLRT) are used to decide if the present solution is significantly better than the foregoing one, as indicated by a $\mathrm{p}$ value $<0.05$. The bootstrapped likelihood ratio test is an additional criterion but was not applicable because this option is not available for analyses with a weighted variable (see below). Another important criterion is the usefulness of the profiles based on theoretical or practical considerations.

LCA can be used for several types of variables like continuous, binary or ordered categorical variables. In our case we used the continuous CIUS score as the only observed dependent variable. Using continuous scores, LCA is often declared as latent profile analysis. We prefer to use the term LCA because it is applicable to all types of variables, including continuous variables.
Normally distributed variables are required for LCA with continuous variables. We applied the $\ln$ (naturalistic log) transformation for CIUS resulting in a skewness of 0.45 and a kurtosis of -0.50 . This $1 n$-transformed variable was standardized (mean 0, SD 1) for interpretational purposes. Differences between classes were tested for the CIUS mean score, hours Internet/week, social participation, generalized trust and age with the Wald $\chi^{2}$ test and these 5 variables defined as auxiliary variables in Mplus. Posterior probabilities were used as weight factors to account for latent class uncertainty.

Calculating proportions of individuals with Internet addiction based on the suggested cut-off for CIUS (28) [19] was used as an additional indicator to judge the latent class solution. When calculating the occurrence of Internet use disorders, all participants who did not report Internet use for private purposes at least $1 \mathrm{~h}$ on a typical weekday or at least $1 \mathrm{~h}$ on a day at the weekend were considered as not having Internet addiction or problematic Internet use.

\section{Weighting Procedures}

Analyses were run using weighted data. Weights were developed separately for the landline sample and mobile-only sample.

The design weights for the landline sample were defined as the inverted product of the inclusion probabilities of each sampling stage, i.e. the community, the household (represented by landline phone numbers) and the target person selection. In accordance with the sampling procedure, the stratification of the communities was considered for the computation of the inclusion probabilities. On the household and person level, the inclusion probability was determined by the number of telephone numbers assigned to each household and the number of target persons living in the respective household. This information was gathered within the telephone interviews. The design weight was standardized to the number of cases in the sample and trimmed at the 2.5 and 97.5 percentile $($ range $0.125-20.689 ; 2.5$ percentile $=0.25 ; 95$ percentile $=$ 2.93). Finally, an iterative proportional fitting algorithm was used to calibrate the design-weighted distribution of socio-economic variables to data of the German population derived from official statistics. The following variables were used for this purpose: age, sex, federal state, size of household, education level (graduation level), unemployment, and migration background.

Analogous to the procedure described above, we constructed the design weights for the mobile-only phone sample. Because we assumed that, in general, a mobile phone number represented one person rather than a household, the number of mobile phone numbers that were assigned to each participant was the only factor considered. The combined design weights for the mobile-only and landline sample were computed by assuming an estimated proportion of $14 \%$ of German residents are exclusively accessible via mobile phone in the 14- to 64-year-old age group. The combined design weights were again standardized, trimmed, and calibrated to the aforementioned characteristics of the German population at ages 14-64 years. The final combined weights ranged from 0.15 to 3.75 .

\section{Results}

\section{Latent Class Analysis}

LCAs with 1-7 classes were performed. The results for each solution are given in table 1 . Lower BIC values and 
Table 1. Latent class models with up to 7 classes

\begin{tabular}{|c|c|c|c|c|c|c|}
\hline LCA & Classes & $\begin{array}{l}\text { Mean CIUS } \\
\text { (z-score) }\end{array}$ & $\mathrm{n}$ & $\begin{array}{l}\text { Posterior } \\
\text { probability }\end{array}$ & $\begin{array}{l}\text { VLMR-LRT } \\
\text { p value }\end{array}$ & $\begin{array}{l}\text { LMR-aLRT } \\
\text { p value }\end{array}$ \\
\hline $\begin{array}{l}\text { Class }=1 \\
\mathrm{BIC}=23.560\end{array}$ & 1 & 0.03 & 8,130 & 1,000 & & \\
\hline $\begin{array}{l}\text { Classes }=2 \\
\mathrm{BIC}=220.945 \\
\text { Entropy }=0.658\end{array}$ & $\begin{array}{l}1 \\
2\end{array}$ & $\begin{array}{r}-0.53 \\
1.13\end{array}$ & $\begin{array}{l}5,564 \\
2,566\end{array}$ & $\begin{array}{l}0.910 \\
0.875\end{array}$ & 0.000 & 0.000 \\
\hline $\begin{array}{l}\text { Classes }=3 \\
\mathrm{BIC}=220.742 \\
\text { Entropy }=0.710\end{array}$ & $\begin{array}{l}1 \\
2 \\
3\end{array}$ & $\begin{array}{r}-0.78 \\
0.52 \\
1.71\end{array}$ & $\begin{array}{l}4,137 \\
2,960 \\
1,033\end{array}$ & $\begin{array}{l}0.904 \\
0.799 \\
0.821\end{array}$ & 0.001 & 0.001 \\
\hline $\begin{array}{l}\text { Classes }=4 \\
\text { BIC }=220.574 \\
\text { Entropy }=0.739\end{array}$ & $\begin{array}{l}1 \\
2 \\
3 \\
4\end{array}$ & $\begin{array}{r}-0.97 \\
0.05 \\
1.07 \\
2.11\end{array}$ & $\begin{array}{r}3,284 \\
2,532 \\
1,841 \\
472\end{array}$ & $\begin{array}{l}0.876 \\
0.819 \\
0.829 \\
0.843\end{array}$ & 0.041 & 0.047 \\
\hline $\begin{array}{l}\text { Classes }=5 \\
\mathrm{BIC}=220.490 \\
\text { Entropy }=0.762\end{array}$ & $\begin{array}{l}1 \\
2 \\
3 \\
4 \\
5\end{array}$ & $\begin{array}{r}-1.04 \\
-0.12 \\
0.77 \\
1.60 \\
2.50\end{array}$ & $\begin{array}{r}2,815 \\
2,448 \\
1,833 \\
871 \\
162\end{array}$ & $\begin{array}{l}0.893 \\
0.820 \\
0.791 \\
0.818 \\
0.847\end{array}$ & 0.153 & 0.162 \\
\hline $\begin{array}{l}\text { Classes }=6 \\
\mathrm{BIC}=220.417 \\
\text { Entropy }=0.769\end{array}$ & $\begin{array}{l}1 \\
2 \\
3 \\
4 \\
5 \\
6\end{array}$ & $\begin{array}{r}-1.16 \\
-0.45 \\
0.22 \\
0.97 \\
1.73 \\
2.59\end{array}$ & $\begin{array}{r}2,271 \\
1,865 \\
1,680 \\
1,410 \\
741 \\
162\end{array}$ & $\begin{array}{l}0.864 \\
0.772 \\
0.802 \\
0.837 \\
0.834 \\
0.849\end{array}$ & 0.253 & 0.264 \\
\hline $\begin{array}{l}\text { Classes }=7 \\
\mathrm{BIC}=220.344 \\
\text { Entropy }=0.791\end{array}$ & $\begin{array}{l}1 \\
2 \\
3 \\
4 \\
5 \\
6 \\
7\end{array}$ & $\begin{array}{r}-1.21 \\
-0.56 \\
0.07 \\
0.71 \\
1.31 \\
1.95 \\
2.72\end{array}$ & $\begin{array}{r}1,777 \\
1,967 \\
1,519 \\
1,288 \\
1,014 \\
446 \\
118\end{array}$ & $\begin{array}{l}0.930 \\
0.775 \\
0.849 \\
0.794 \\
0.767 \\
0.845 \\
0.860\end{array}$ & 0.211 & 0.220 \\
\hline
\end{tabular}

higher entropy measures (both as desired) can be found with the increasing number of classes. Non-significant $p$ values $(>0.05)$ of VLMR-LRT and LMR-aLRT indicate that models 5-7 are each not better than the foregoing one. In the 5-class model and the 6-class model, the same extreme group with high CIUS score emerges $(n=162)$ speaking in favour of these models. From the 4-class model onwards, in each solution, two groups have distinctively higher CIUS scores than the other classes as hypothesized (supposed to represent the at-risk group and the addiction group). An 8-class solution was not considered because a group of $n=8$ emerged as a part of the group of $\mathrm{n}=118$ from the 7 -class solution. Taking all the information into account, the 6-class model was chosen and explored further because the same number $(n=162)$ of extreme cases occurs as in the foregoing model and BIC and entropy speak in favour of the 6 classes compared to the 5 classes.

The 6 classes were compared with respect to CIUS mean scores, time spent in the Internet, social participation and trust (table 2). The LCA resulted in 6 mutual exclusive classes with respect to CIUS mean scores. In all variables, classes differed significantly with class 5 and 6 showing higher CIUS scores, more time spent in the Internet and less social participation. Differences are more pronounced for class 6 throughout. With respect to generalized trust, only class 6 shows lower scores. Regarding age, class 5 is the youngest group. Data can be interpreted as class 5 representing at-risk use and class 6 Internet addiction. The observed range of CIUS-mean scores for the 
Table 2. Characteristics of the 6-class model

\begin{tabular}{|c|c|c|c|c|c|}
\hline 1 & $1.36(0.04)$ & $8.73(0.22)$ & $5.70(0.06)$ & $2.63(0.02)$ & $40.60(0.32)$ \\
\hline 2 & $5.08(0.06)$ & $11.25(0.33)$ & $5.75(0.07)$ & $2.70(0.02)$ & $36.90(0.40)$ \\
\hline 3 & $9.41(0.07)$ & $13.10(0.34)$ & $5.65(0.07)$ & $2.70(0.02)$ & $33.91(0.40)$ \\
\hline 4 & $15.41(0.10)$ & $16.08(0.41)$ & $5.51(0.08)$ & $2.72(0.02)$ & $31.08(0.38)$ \\
\hline Significance, $\mathrm{p}^{*}$ & $<0.001$ & $<0.001$ & $<0.001$ & $<0.001$ & $<0.001$ \\
\hline
\end{tabular}

* Wald $\chi^{2}$ test based on weighted data and class uncertainty with posterior probabilities.

Table 3. Occurrence of Internet addiction (class 6) and problematic Internet use (class 5) in different age groups

\begin{tabular}{|c|c|c|c|c|c|c|}
\hline \multirow[t]{2}{*}{ Age } & \multicolumn{3}{|c|}{ Internet addiction, \% (95\% CI) } & \multicolumn{3}{|c|}{ At-risk Internet use, \% (95\% CI) } \\
\hline & total & female & male & total & female & male \\
\hline $14-24(\mathrm{n}=2,937)$ & $2.4(1.9-3.1)$ & $2.4(1.6-3.5)$ & $2.5(1.7-3.5)$ & $13.6(12.4-14.8)$ & $14.8(13.0-16.8)$ & $12.4(10.4-14.7)$ \\
\hline $14-16(n=693)$ & $4.0(2.7-5.7)$ & $4.9(2.8-8.5)$ & $3.1(1.8-5.3)$ & $15.4(12.8-18.5)$ & $17.2(13.2-22.2)$ & $13.7(10.5-17.7)$ \\
\hline
\end{tabular}

6 classes were respectively $0-3$ (class 1 ), 4-7 (class 2 ), 8-12 (class 3), 13-19 (class 4), 20-29 (class 5) and 30-52 (class 6).

Participants with probable Internet addiction spent $4.2 \mathrm{~h} /$ day in the Internet for private purposes and those with at-risk use spent $3.1 \mathrm{~h} /$ day.

\section{Occurrence of Latent Classes}

As shown in table 3,1.0\% of the total sample belongs to class 6 (probable Internet addiction) and $4.6 \%$ to class 5 (probable at-risk Internet use). In younger age groups, proportions of both groups are higher. Looking at probable Internet addiction in the entire sample and in subgroups according to age, no significant differences between males and females can be found: age 18-64 $\left(\chi^{2}=\right.$ 2.33 ; d.f. $=1$; design d.f. $=48 ; \mathrm{p}=0.13)$, age $14-24\left(\chi^{2}=\right.$ 0.004 ; d.f. $=1$; design d.f. $=48 ; \mathrm{p}=0.95)$, and age $14-16$ $\left(\chi^{2}=1.02 ;\right.$ d.f. $=1$; design d.f. $\left.=48 ; \mathrm{p}=0.32\right)$.

Looking at probable problematic Internet use in the entire sample and in different age groups, proportions again do not differ significantly according to gender: age $18-64\left(\chi^{2}=1.31\right.$; d.f. $=1$; design d.f. $\left.=48 ; \mathrm{p}=0.27\right)$, age 14-24 $\left(\chi^{2}=1.90\right.$; d.f. $=1$; design d.f. $\left.=48 ; \mathrm{p}=0.17\right)$, and age $14-16\left(\chi^{2}=1.02\right.$; d.f. $=1$; design d.f. $\left.=48 ; \mathrm{p}=0.32\right)$.
The proportion for probable Internet addiction was markedly higher in subjects recruited through mobileonly $(1.95 \%)$ compared to landline numbers $\left(0.86 \% ; \chi^{2}=\right.$ 55.74; d.f. $=1$; design d.f. $=48 ; \mathrm{p}<0.001)$. Looking at the main activity in the Internet, female class- 6 members used online games less often, however this was only a tendency and not statistically significant ( 7.2 vs. $33.6 \% ; \chi^{2}=$ 3.79 ; d.f. $=1$; design d.f. $=48 ; \mathrm{p}=0.057)$. We conducted a logistic regression analysis (method: enter) with class 6 as dependent variable and included gender, age, migration background and unemployment (Nagelkerke's pseudo $\mathrm{R}^{2}$ was 0.091 ). Age, migration background and unemployment were significantly related with Internet addiction; odds ratios $(95 \% \mathrm{CI})$ were for age 0.95 (0.94-0.97), migration background 3.04 (2.12-4.36), and unemployment 3.13 (1.74-5.65). More males than females were in class 6 , however this was not statistically significant in the regression model (OR 1.53; 0.99-2.35). The addicted group differed significantly from the others in all CIUS items. Within this subgroup, the means of the CIUS items were quite similar with the lowest mean (2.90; SE 0.11) in item 11 [How often do you neglect your daily obligations (work, school, or family life) because you prefer to go on the Internet?] and the highest (3.76; SE 0.10) in item 3 
[How often do others (e.g., partner, children, parents) say you should use the Internet less?].

For comparison, the recommended cut-off (28) for the CIUS was used to estimate occurrence of Internet addiction resulting in $1.5 \%$ (CI 1.3-1.7) for the entire sample, $1.3 \%$ (CI 1.0-1.7) for females, and 1.7\% (CI 1.3-2.1) for males. Based on participants aged 14-24, Internet addiction occurred in 3.8\% (CI 3.0-4.6) in total, 4.5\% (CI 3.36.0 ) in females, and 3.0\% (CI 2.2-4.3) in males. Based on participants aged 14-16, Internet addiction occurred in 6.3\% (CI 4.6-8.4) in total, 8.6\% (CI 5.5-13.0) among females, and $4.1 \%$ (CI 2.6-6.3) among males.

\section{Discussion}

This is the largest representative general population study that aimed to quantify the occurrence of Internet addiction and describe related characteristics so far. Only two studies on prevalence estimates have used a general population approach. In face of lacking generally accepted diagnostic criteria to define Internet addiction, this study used a different approach by analysing latent classes. These classes were based on the CIUS, a questionnaire which covers well-accepted signs of problematic Internet use. A 6-class solution showed the best fit and included 2 classes that differed in variables that are related to problematic and addictive Internet use compared to the other groups. One group showed highest rates in CIUS scores and time spent in the Internet, whereas social participation was lowest. This was the only group differing in social trust compared to all other groups and was considered as representing the subgroup of those likely to have Internet addiction. A second group shows the second highest values in CIUS scores and time spent in the Internet, the second lowest scores in social participation and the lowest mean age. This class was considered as probably having at-risk Internet use.

The differences with respect to social participation are an important finding showing that the extensive use of the Internet may have led to withdrawal from life in society. This could be observed in both groups but was strongest in class 6 representing Internet addiction. Proposed criteria for Internet addiction by Tao et al. [7] include a course criterion requiring at least 3 months of duration and $6 \mathrm{~h}$ of Internet non-business usage per day. In our study, participants with probable problematic Internet use were online for private purposes approximately $3 \mathrm{~h} /$ day, those with probable Internet addiction about $4 \mathrm{~h}$. Our findings shed some light on defining a minimum time spent in the Internet as criterion for Internet addic- tion. According to our data, $6 \mathrm{~h}$ as a threshold are too high. It might be discussed if such a criterion is useful at all since it would exclude those who try to reduce time spent in the Internet but still are addicted to it.

Using the class found as an estimator for the occurrence of Internet addiction results in a total proportion of $1 \%$ for all participants aged 14-64. This is lower than in findings of several prevalence studies but quite comparable to those using a general population approach $[10,11]$. In younger age groups, proportions are - as expected - higher with up to $4 \%$ for Internet addiction in the 14- to 16-year-old participants. Most studies so far have found prevalence estimates to be higher in males than in females [e.g. 11,25, 26]. This is not true in our study. In all age groups looked at, no statistical difference could be found according to gender. This underlines other findings pointing at gender to be no predictor of Internet addiction [27]. Moreover, in the youngest group (14-16) of our study, proportions were highest for females, although not statistically significant. Interestingly, female participants showed a tendency to use less often online games as their main activity on the Internet.

Some limitations of our study have to be mentioned when interpreting its results: the inclusion criterion for administering the CIUS was use of the Internet for private purposes at least $1 \mathrm{~h}$ on a typical weekday or at least $1 \mathrm{~h}$ on a day at the weekend. All participants with less time spent in the Internet were considered as not having problematic use or Internet addiction. It is not very likely that this filter resulted in biased findings, however it cannot be fully ruled out. Another possible shortcoming might be that the CIUS which served as the basis for the LCA might not cover all relevant aspects to specify Internet addiction. However, salience, withdrawal, loss of control, conflict and coping with unpleasant mood are included and resemble current suggestions for classifying Internet use disorders. Another crucial point is that the identification of the respective groups depends on the latent class model that was considered to fit data best. For example, if the decision had been that the 4-class model would fit data best, the rate of Internet addiction would have been nearly threefold. However, finding an identical number of cases $(n=162)$ in the 5 - and 6-class-model gives strong evidence for the estimates based on the model we chose. In addition, using the suggested cut-off of 28 for CIUS might serve as another indicator. This results in a rate of $1.5 \%$ positive cases and strengthens our solution.

A clear advantage of this study is the large and representative sample size. In addition, including mobile-only participants reduces a possible sample selection bias. Findings show that the proportion of probable Internet 
addiction in the mobile-only sample doubles that of the landline sample. Therefore, the inclusion of mobile-only participants is of special importance. In addition, the LCA approach is currently a viable method considering that no consensus on criteria to define Internet addiction or problematic Internet use is available.

We conclude that data presented here give a reliable basis to estimate the magnitude of problems following excessive Internet use. High proportions in young age groups give reasons for preventive measures and for further research. This is especially important since use and misuse of mobile phones is prevalent in adolescents [28] and the technical possibilities of current smartphone technology might be seen as a risk for increasing prevalence rates. In order to fully grasp the significance of our data and those of other studies, it is crucial to assess whether participants who were suggested to have Internet addiction are really significantly impaired in a clinical sense as claimed in proposals for classifying Internet addiction $[7,16]$. In addition, longitudinal research can be seen as a necessity to understand the nature and course of Internet addiction. Moreover, Internet addiction has been found to be related to other behavioural addictions [27] and psychiatric comorbidity $[29,30]$. This was not assessed in our study and should be part of future general population-based surveys. Elevated rates of Internet addiction in unemployed participants give reason for focusing on this group when considering to offer help. The same is true for individuals with migration background. Unemployment centres might serve as a suitable site for screening and brief interventions.

\section{References}

$>1$ Block JJ: Issues for DSM-V: Internet addic- 13 Beard KW: Internet addiction: a review of tion. Am J Psychiatry 2008;165:306-307.

2 American Psychiatric Association: Diagnostic and Statistical Manual of Mental Disorders, ed 5. Washington, APA, 2013.

3 Meerkerk GJ, Van den Eijnden R, Garretsen HFL: Predicting compulsive Internet use: it's all about sex! Cyberpsychol Behav 2006;9:95-103.

4 Van Rooij AJ, Schoenmakers TM, van de Eijnden $\mathrm{R}$, van de Mheen D: Compulsive Internet use: the role of online gaming and other Internet applications. J Adolesc Health 2010;47:51-57.

5 Kuss DJ, Griffiths MD: Online social networking and addiction - a review of the psychological literature. Int J Environ Res Public Health 2011;8:3528-3552.

6 Rehbein F, Mössle T: Video game and Internet addiction: is there a need for differentiation? Sucht 2013;59:129-142.

$>7$ Tao R, Huang XQ, Wang JN, Zhang HM, Zhang Y, Li MC: Proposed diagnostic criteria for Internet addiction. Addiction 2010;105 556-564.

8 Christakis DA: Internet addiction: a 21st century epidemic? BMC Med 2010;8:3.

$\checkmark$ Cao H, Sun Y, Wan YH, Hao JH, Tao FB: Problematic Internet use in chinese adolescents and its relation to psychosomatic symptoms and life satisfaction. BMC Public Health 2011;11:28.

$>10$ Aboujaoude E, Koran LM, Gamel N, Large MD, Serpe RT: Potential markers for problematic Internet use: a telephone survey of 2,513 adults. CNS Spectr 2006;11:750-755.

$>11$ Bakken IJ, Wenzel HG, Gotestam KG, Johansson $\mathrm{A}$, Oren $\mathrm{A}$ : Internet addiction among Norwegian adults: a stratified probability sample study. Scand J Psychol 2009;50:121-127.

$>12$ Young KS: Internet addiction: the emergence of a new clinical disorder. Cyberpsychol Behav 1998; 1:237-244. current assessment techniques and potential assessment questions. Cyberpsychol Behav 2005;8:7-14.

14 Young KS: Caught in the Net: How to Recognize the Signs of Internet Addiction - and a Winning Strategy for Recovery. New York, Wiley, 1998.

15 Chen SH, Weng LC, Su YJ, Wu HM, Yang PF: Development of Chinese Internet Addiction Scale and its psychometric study. Chin J Psychol 2003;45:279-294

16 Ko CH, Yen JY, Chen SH, Yang MJ, Lin HC, Yen CF: Proposed diagnostic criteria and the screening and diagnosing tool of Internet addiction in college students. Compr Psychiatry 2009;50:378-384.

17 Vermunt JK, Magidson J: Latent class cluster analysis; in Hagenaars JA, McCutcheon AL (eds): Applied Latent Class Analysis. Cambridge, University Press, 2002, pp 89-106.

18 Van Rooij AJ, Schoenmakers TM, Vermulst AA, Van den Eijnden RJ, Van de Mheen D: Online video game addiction: identification of addicted adolescent gamers. Addiction 2011;106:205-212.

19 Meerkerk GJ, Van Den Eijnden R, Vermulst AA, Garretsen HFL: The Compulsive Internet Use Scale (CIUS): some psychometric properties. Cyberpsychol Behav 2009;12:1-6.

20 European Commission: Special eurobarometer 362: E-communication household survey. http://eceuropaeu/public_opinion/archives/ ebs/ebs_362_enpdf 2011 (accessed February 2013).

21 Lindstrom M: Social capital, the miniaturisation of community and self-reported global and psychological health. Soc Sci Med 2004; 59:595-607.

22 Hanson BS, Östergren PO, Elmstahl S, Isaacsson SO, Ranstam J: Reliability and validity assessments of measures of social networks, social support and control - results from the
Malmö Shoulder and Neck Study. Scand J Public Health 1997;25:249-257.

23 Muthén LK, Muthén BO: Mplus User's Guide, ed 5. Los Angeles, Muthèn \& Muthén, 1998.

24 Nylund KL, Asparouhov TM, Muthén BO: Deciding on the number of classes in latent class analysis and growth mixture modeling: a Monte Carlo simulation study. Struct Equ Modeling 2007;14:535-569.

25 Kormas G, Critselis E, Janikian M, Kafetzis D, Tsitsika A: Risk factors and psychosocial characteristics of potential problematic and problematic Internet use among adolescents: a crosssectional study. BMC Public Health 2011;11:8.

26 Tsai HF, Cheng SH, Yeh TL, Shih CC, Chen KC, Yang YC, Yang YK: The risk factors of Internet addiction - a survey of university freshmen. Psychiatry Res 2009;167:294-299.

27 Villella C, Martinotti G, Di Nicola M, Cassano M, La Torre G, Gliubizzi MD, Messeri I, Petruccelli F, Bria P, Janiri L, Conte G: Behavioural addictions in adolescents and young adults: results from a prevalence study. J Gambl Stud 2011;27:203-214.

28 Martinotti G, Villella C, Di Thiene D, Di Nicola M, Bria P, Conte G, Cassano M, Petruccelli F, Corvasce N, Janiri L, La Torre G: Problematic mobile phone use in adolescence: A cross-sectional study. J Public Health 2011;19:545-551.

29 Di Nicola M, Tedeschi D, Mazza M, Martinotti G, Harnic D, Catalano V, Bruschi A, Pozzi G, Bria P, Janiri L: Behavioural addictions in bipolar disorder patients: role of impulsivity and personality dimensions. J Affect Disord 2010;125:82-88.

30 Carli V, Durkee T, Wasserman D, Hadlaczky G, Despalins R, Kramarz E, Wasserman C, Sarchiapone M, Hoven CW, Brunner R, Kaess M: The association between pathological Internet use and comorbid psychopathology: a systematic review. Psychopathology 2013;46:1-13. 